\title{
Amiodarone Improper Rapid Intravenous Injection Can Be Ended with Serious Complications and Even Death
}

\author{
Hasan Ali Farhan ${ }^{1}$ and Israa Fadhil Yaseen ${ }^{2}$ \\ 1.Department of Cardiology, Baghdad Teaching Hospital-Medical City, Iraqi Board for Medical Specializations, Scientific Council \\ of Cardiology, Baghdad 00964, Iraq \\ 2.Department of Cardiology, Baghdad Teaching Hospital-Medical City, Iraqi Board for Medical Specializations, Scientific Council \\ of Clinical Pharmacy, Baghdad 00964, Iraq
}

\begin{abstract}
Amiodarone is one of the widely used antiarrhythmic drugs related to class III, used in the treatment of SVT (supraventricular) and ventricular arrhythmias. However, improper use of this drug such as pushing high concentration with rapid injection can increase the rate of adverse effects. The most common adverse effects associated with intravenous amiodarone are hypotension (16\%), heart block or bradycardia (4.9\%), and peripheral phlebitis (8-55\%), this can be treated by diluting the drug and slowing the rate of infusion or discontinuing the drug. In addition, hypotension can be refractory and lead to a fatal outcome and death. Because phlebitis may occur, the drug should be given through a central line when possible. Some literatures refer to the administration of intravenous amiodarone in the treatment of arrhythmias such as in SVT, as "given over 10 minute" without focusing on the dilution and rate of infusion, therefore many residents give it with rapid direct intravenous injection without dilution and even during less than 10 minutes. From currently daily practice we are reporting two cases of amiodarone improper administration, first case "amiodarone-associated refractory hypotension" and second case "amiodarone-associated death".
\end{abstract}

Key words: Amiodarone, hypotension, phlebitis, pulseless electrical activity, CCU.

\section{Introduction}

Amiodarone is class III antiarrhythmic drug widely used in the treatment of arrhythmias. Amiodarone administration is associated with well known long term adverse effects on the lung, thyroid gland, and liver, as well as, with immediate adverse effects like phlebitis, refractory hypotension, shock, and/or even death. The current paper will highlight the immediate adverse effects which are resulting from improper parenteral administration of amiodarone, based on clinical practice cases. Such adverse effects can be avoided by expanding knowledge about the precise rate and duration of amiodarone parenteral administration, in addition to hemodynamic monitoring of the patient during infusion.

Corresponding author: Israa Fadhil Yaseen, bachelor, research field: cardiology. E-mail: pharm18i@yahoo.com.

\section{Case (1)}

A 32-year-old woman with gestational diabetes, presented to the internal ward two weeks postpartum (caesarian section) with dry cough, generalized edema, dyspnea, and palpitation for five days, without fever and had a good urine output. Her BP (blood pressure) was $100 / 76 \mathrm{~mm} \mathrm{Hg}$ with a PR (pulse rate) $110 \mathrm{bpm}$ (beat per minute).

Results of her investigations were: blood urea 40 $\mathrm{mg} / \mathrm{dl}$, serum creatinine $0.8 \mathrm{mg} / \mathrm{dl}$, fasting blood sugar $199 \mathrm{mg} / \mathrm{dl}$, chest x-ray showed globular heart with clear lung field, and ECG (electrocardiograph) showed SVT, therefore the patient referred from the internal ward to the CCU (cardiac care unit).

At CCU the patient had a peripheral cannula (near the wrist) through which a loading dose of amiodarone $(300 \mathrm{mg}$ ) was pushing without dilution over less than 10 minutes (about 3-4 minutes), during administration 
the patient was shouting and the medical staff thought that there is an extravasation due to the redness of skin area around the cannula, so they stopped the infusion, fixed a new cannula at the median cubital vein, adding another $150 \mathrm{mg}$ amiodarone to the remaining amiodarone in the syringe as they thought that some of the drug had been extravasated, and gave it to the patient, but again during injection the patient was shouting then the BP declined to 50/20 $\mathrm{mm} \mathrm{Hg}$ and a PEA (pulseless electrical activity) had been developed. See the Fig. 1. DC shock was given to her twice then adenosine $6 \mathrm{mg}$ rapid IV followed by $12 \mathrm{mg}$, and finally Verapamil $10 \mathrm{mg}$ IV was given to the patient.

\section{Case (2)}

A 63-year-old man has a history of ischemic heart disease, hypertension, diabetes, and $\mathrm{x}$-smoker. Presented to the emergency room with disturb level of consciousness and palpitation with a history of progressive dyspnea and orthopnea for two days, his ECG revealed paroxysmal AF (atrial fibrillation), and his chest x-ray showed pulmonary edema. The patient referred to CCU.

At CCU, the patient was confused, agitated, dyspnic, tachypnic, with bilateral basal lung crepitations, and has a decrease in urine output, with BP 130/70 mm Hg and PR 165 bpm.

Other results of his investigations were: random blood sugar $148 \mathrm{mg} / \mathrm{dl}$, blood urea $131 \mathrm{mg} / \mathrm{dl}$, serum creatinine $3.2 \mathrm{mg} / \mathrm{dl}$, serum potassium $3.4 \mathrm{mEq} / \mathrm{L}$.

He was treated with undiluted amiodarone $300 \mathrm{mg}$ rapid direct injection in the peripheral vein over less than 10 minutes, then immediately his BP started to decrease to $75 / 50 \mathrm{~mm} \mathrm{Hg}$, his PR on monitor was 120 pbm (sinus rhythm) but without pulses (PEA).

Amiodarone stopped and started dopamine 20 microgram $/ \mathrm{kg} / \mathrm{min}$ to be given intravenously over 24 hours.

After 9 hours the patient developed apnea, cyanosis, undetectable pulse and BP despite sinus rhythm on the monitor, then patient died.

\section{Discussion}

Amiodarone is one of the well known irritant drugs of blood vessels and the reported rates of phlebitis associated with it ranging from $8 \%$ to $54.5 \%$. Also it has been reported that phlebitis rates can be as high as $25 \%$ when concentrations greater than $1.5 \mathrm{mg} / \mathrm{mL}$ are used $[1,2]$. In general, phlebitis associated with amiodarone occurred at doses more than $3 \mathrm{mg} / \mathrm{mL}$ and it can be minimized by using a concentration of 2.5

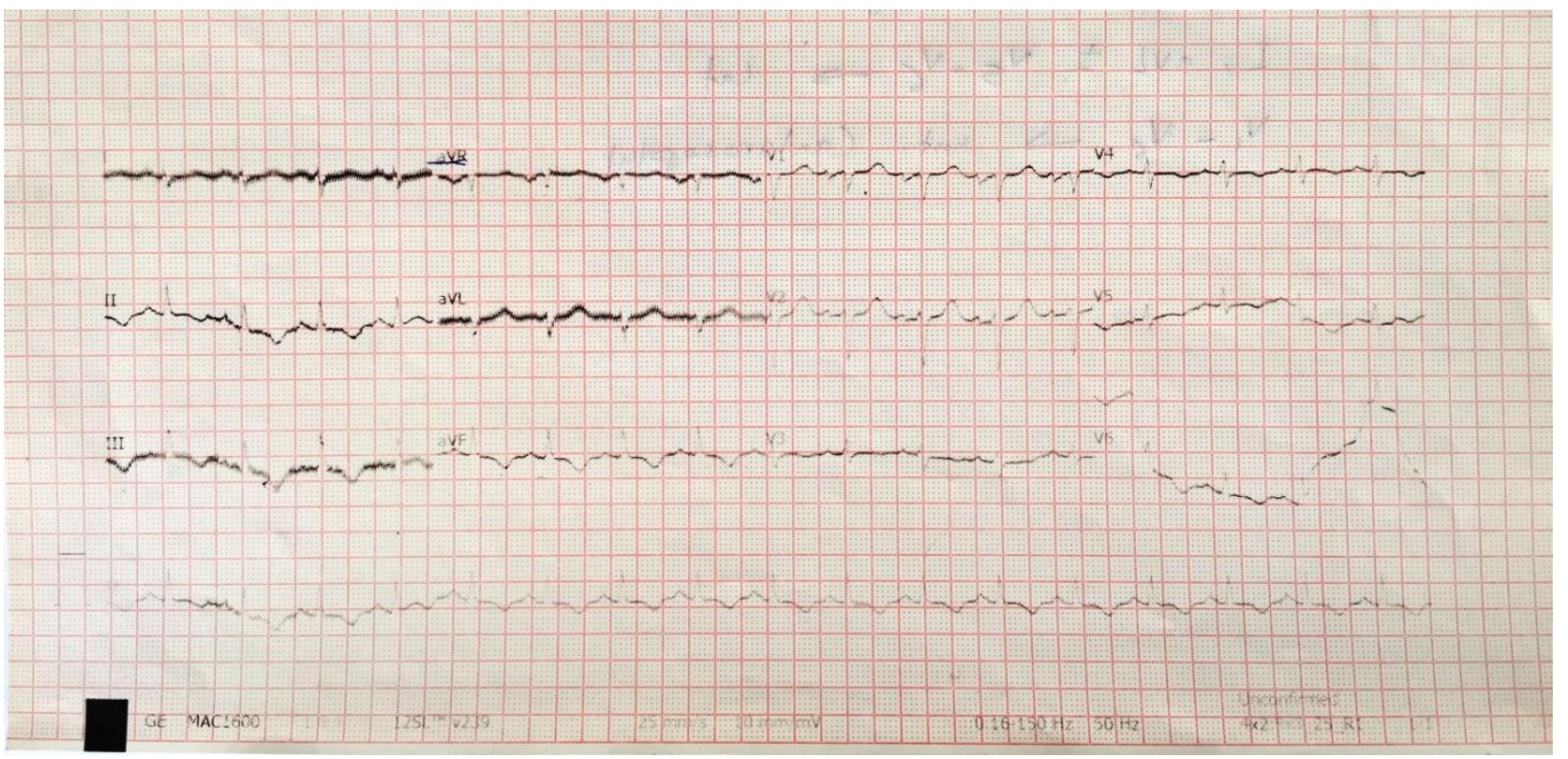

Fig. 1 ECG of 32 year-old-woman with SVT after receiving rapid injection and high concentration of amiodarone. 
$\mathrm{mg} / \mathrm{mL}$ and in-line filters. When a first sign of phlebitis appears, the infusion should be stopped with changing the infusion site to the unaffected arm [3]. For all concentrations of amiodarone, a central venous catheter is recommended and it is required for concentrations greater than $2 \mathrm{mg} / \mathrm{mL}$, in addition the rate of infusion should not exceed $30 \mathrm{mg} / \mathrm{min}$ [4]. In case (1), the patient received more than $70 \mathrm{mg} / \mathrm{min}$ of amiodarone and this was the cause of her shouting and redness of the affected area during amiodarone injection. Therefore, amiodarone should be diluted before administration (e.g. $300 \mathrm{mg}$ diluted in $20-30 \mathrm{~mL}$ D5W (5\% dextrose in water) in case of cardiac arrest [5] or $150 \mathrm{mg}$ in $100 \mathrm{~mL} \mathrm{D5W}$ in VT (ventricular tachycardia); or normal saline also can be used as a diluent for Nexterone [4]).

Other adverse effects related to rapid injection of amiodarone are hypotension, bradycardia, and PEA which are so clearly found in both cases presented in this paper. PEA which is a clinical situation exists when organized electrical activity (not a specific dysrrhythmia, other than VT) is appeared on the cardiac monitor, during which the patient is nonresponder and not breathing, in addition the pulse cannot be felt. The rhythm can be shown as a sinus tachycardia as appeared in the Fig. 1 [6]. Regarding case (2), death may be related to refractory hypotension [7] which did not respond to dopamine infusion. In addition, hepatocellular necrosis, acute renal failure, and death are associated with the use of high concentration of the drug and increased rate of infusion [4] and as it has been mentioned above, this patient had already elevated blood urea and serum creatinine.

\section{Conclusion}

Up to our knowledge and literatures review, we didn't find reported cases of amiodarone associated death and improper administration of this widely used antiarrhythmic drug which may end with serious complications and even death. Therefore, focus and orientation on the proper IV administration of such drug is required in order to bridge this practice gap.

\section{References}

[1] Siddoway, L. A. 2003. "Amiodarone: Guidelines for Use and Monitoring.” Am. Fam. Physician. 68: 2194.

[2] Norton, L., Ottoboni, L. K., Varady, A., Yang-Lu, C-Y., Becker, N., Cotter, T., Pummer, E., Haynes, A., Forsey, L., Matsuda, K., and Wang, P. 2013. "Phlebitis in Amiodarone Administration: Incidence, Contributing Factors, and Clinical Implications." American Journal of Critical Care 22: 499.

[3] Brady Boyce, B. A., and Homer Yee, B. 2012. "Incidence and Severity of Phlebitis in Patients Receiving Peripherally Infused Amiodarone." Critical Care Nurse 32: 27-34.

[4] Gahart, B. L., and Nazareno, A. R., eds. 2015. Intravenous Medications: A Handbook for Nurses and Health Professionals. Mosby: United States of America.

[5] White, C. M., Song, J. C., and Kalus, J. S. 2013. "Cardiac Arrhythmias". In Koda-Kimble and Young's applied therapeutics : The clinical use of drugs, edited by Alldredge, B. K., Corelli, R. L., Ernst, M. E., Guglielmo, B. J., Jacobson, P. A., Kradjan, W. A., Williams, B. R. 10th ed. Philadelphia, PA: Lippincott Williams \& Wilkins.

[6] Aehlert, B., ed. 2012. ACLS Study Guide. Mosby: United States of America.

[7] NEXTERONE [package insert]. 2011. Bloomington, IN: Baxter Pharmaceutical Solutions, LLC. 